\title{
CSF sulfatide distinguishes between normal pressure hydrocephalus and subcortical arteriosclerotic encephalopathy
}

M Tullberg, J-E Månsson, P Fredman, A Lekman, K Blennow, R Ekman, L E Rosengren, M Tisell, C Wikkelsø

\begin{abstract}
Objectives-To examine the CSF concentrations of molecules reflecting demyelination, neuronal and axonal degeneration, gliosis, monoaminergic neuronal function, and aminergic and peptidergic neurotransmission in a large series of patients with normal pressure hydrocephalus (NPH) or subcortical arteriosclerotic encephalopathy (SAE), to elucidate pathogenic, diagnostic, and prognostic features. Methods-CSF concentrations of glycosphingolipid (sulfatide), proteins (neurofilament triplet protein (NFL), glial fibrillary acidic protein (GFAP)), neuropeptides (vasoactive intestinal peptide (VIP), 4-aminobutyric acid (GABA)), and monoamines (homovanillic acid (HVA), 5-hydroxy-indoleacetic acid (5-HIAA), 4-hydroxy-3-methoxyphenylglycol (HMPG)) were analysed in 43 patients with NPH and 19 patients with SAE. The diagnoses of NPH and SAE were based on strict criteria and patients with NPH were subsequently operated on. Twelve clinical variables, psychometric tests measuring perceptual speed, accuracy, learning, and memory and a psychiatric evaluation were performed in all patients and before and after a shunt operation in patients with NPH.
\end{abstract}

Results-The CSF sulfatide concentration was markedly increased in patients with SAE (mean 766, range 300-3800 $\mathrm{nmol} / \mathrm{l}$ ) compared with patients with NPH (mean 206 , range $50-400 \mathrm{nmol} / 1) \quad(\mathrm{p}<0.001)$. 5-HIAA, GABA, and VIP in CSF were higher in patients with SAE than in patients with NPH. The patients with NPH with cerebrovascular aetiology had higher sulfatide concentrations and a poorer outcome after shunt surgery than patients with NPH with other aetiologies.

Conclusions-The pathogenesis of the white matter changes in NPH and SAE is different and ischaemic white matter changes can be a part of the NPH state. The markedly increased CSF sulfatide concentrations in patients with SAE indicate ongoing demyelination as an important pathophysiological feature of SAE. The CSF sulfatide concentration distinguished between patients with SAE and those with NPH with a sensitivity of $74 \%$ and a specificity of $94 \%$, making it an important diagnostic marker.

(F Neurol Neurosurg Psychiatry 2000;69:74-81)
Keywords: normal pressure hydrocephalus; subcortical arteriosclerotic encephalopathy; sulfatide

Normal pressure hydrocephalus (NPH) is characterised by gait disturbance, mental deterioration, and urinary incontinence in patients with an enlarged ventricular system and a normal CSF pressure. ${ }^{12}$ An important pathophysiological feature of NPH is a dysfunction of CSF dynamics with reduced absorption through the arachnoid villi, a compensatory CSF flow into the periventricular white matter, and a transcapillary CSF resorption. ${ }^{3-6}$ The periventricular tissue is characterised by disruption of the ependyma, oedema, neuronal degeneration, and gliosis. ${ }^{578} \mathrm{On}$ MRI, white matter changes appear as periventricular confluent lesions, most pronounced around the frontal horns. ${ }^{9-12}$

Patients with subcortical arteriosclerotic encephalopathy (SAE) often present with an enlarged ventricular system and symptoms and signs similar to those seen in NPH. ${ }^{13-15}$ Neuropathologically, SAE is characterised by a pronounced obliterative microangiopathy of the cerebral deep white matter, ${ }^{16}{ }^{17}$ with extensive demyelination, lacunae, and widespread symmetric degeneration. ${ }^{18}$

Characteristic MRI changes in SAE, besides enlarged ventricles, are patchy periventricular hyperintensities representing deep white matter infarctions, ${ }^{141519}$ sometimes difficult to differentiate from the white matter changes seen in $\mathrm{NPH}$.

Thus, patients with NPH or SAE can present with similar symptoms and signs and morphological changes at MRI, and the two entities can probably also coexist in a single patient, ${ }^{21}$ with diagnostic difficulties as a consequence. The diagnostic investigation of patients with NPH and those with SAE and the selection of candidates for shunt surgery is sometimes difficult and requires extensive investigations including radiology, CSF dynamic measurements, and CSF biochemical analyses. Despite extensive and invasive investigations, the success rate of surgery in NPH in many centres is not higher than $70 \%$. Additional knowledge of the deep white matter changes, including the ongoing pathophysiological processes, might elucidate pathogenic and diagnostic features of these diseases.

The CSF reflects ongoing metabolic processes in the brain parenchyma including the white matter, capturing rapid changes in the microenvironment at a cellular level. The CSF neurofilament triplet protein (NFL) concentration is greater in patients with NPH than in 
healthy controls. ${ }^{22} \mathrm{NFL}$ is higher in patients with an excellent outcome of shunt surgery than in those with a poor shunt effect, indicating that the symptoms in the patients with $\mathrm{NPH}$ with high NFL are associated with an ongoing but reversible axonal degeneration. Demyelination as a characteristic pathophysiological feature of $\mathrm{NPH}$ was suggested by Sutton et al, who found increased immunoreactive myelin basic protein in $80 \%$ of patients with $\mathrm{NPH} .{ }^{23}$

In patients with vascular dementia and demyelination, blood-brain barrier damage is often seen, ${ }^{24}$ whereas the barrier is intact in most patients with $\mathrm{NPH}$, except those with cerebrovascular aetiology. ${ }^{25}$

The purpose of the present study was to examine the CSF concentrations of molecules reflecting demyelination, neuronal and axonal degeneration, gliosis, monoaminergic neuronal function, and aminergic and peptidergic neurotransmission in a large series of NPH and patients with SAE, to elucidate pathogenic, diagnostic, and prognostic features.

\section{Materials and methods}

PATIENTS

Sixty five consecutive patients diagnosed as having NPH and 25 as having SAE at the Department of Neurology, Sahlgren's University Hospital, between December 1994 and January 1997 were primarily included. Twenty two patients with NPH and six patients with SAE who refused lumbar puncture or for other reasons did not have a CSF examination were excluded, leaving 43 patients with $\mathrm{NPH}$ and 19 with SAE in the study. The patients with NPH subsequently received a ventriculoperitoneal shunt (a Sophy programmable pressure valve (Sophysa, Orsay Cedex, France) or a Delta Valve (Medtronic PS Medical, Santa Barbara, USA)) or were subjected to endoscopic third ventriculostomy $(n=3)$.

The diagnosis of NPH was based on the presence of the following: (a) gait disturbance; (b) mental deterioration, or urinary incontinence, or both; (c) enlarged ventricles on CT or MRI with an Evans index (maximal width of frontal horns/maximal width of inner skull)>0.30; (d) a lumbar CSF pressure below $20 \mathrm{~cm} \mathrm{H}_{2} \mathrm{O}$; (e) ventricular filling and block of convexity flow on radionuclide cisternography in all patients except those with aqueductal stenosis. ${ }^{26}$ In some patients fulfilling these criteria, but with signs of other disorders, such as vascular lesions, the CSF tap test, ${ }^{27}$ and regional cerebral blood flow $(\mathrm{rCBF}){ }^{28}$ measurement were performed to strengthen the indication for surgery. Only patients with improvement at the CSF tap test and a characteristic pattern on $\mathrm{rCBF}^{28} 29$ were considered eligible for shunt surgery.

The diagnostic criteria for SAE were all of the following: (a) mental deterioration (predominantly astenoemotional disorder and frontal cognitive dysfunction); (b) gait disturbance (ataxia or motor dysfunction of subcortical type); (c) focal neurological signs; (d) vascular risk factors such as hypertension and diabetes, or presence of systemic vascular disease; (e) bilateral and multiple or diffuse subcortical-paraventricular deep white matter changes on MRI or CT ( $>2 \times 2 \mathrm{~mm})$, lacunar infarctions, an enlarged ventricular system, and absence of more than one cortical infarction (figure 1). The criteria are compatible with those suggested by others. ${ }^{14}$ Fifteen of the patients with SAE had no cortical infarction, the remaining four had one cortical infarction.

The patients with NPH (30 men and 13 women) were 66 (12) years old (mean (SD)) (range 19 to 80 years). Mean height was 173 (SD 11) $\mathrm{cm}$ and weight 78 (SD 17) $\mathrm{kg}$. The mean duration of symptoms was 28 (SD 29) months, and the primary cause of NPH was subarachnoid haemorrhage in $21 \%$ of the patients, trauma in $9 \%$, cerebrovascular disorder in $12 \%$, and idiopathic origin in $49 \%$. Further probable causes of NPH were meningeoma $(n=1)$, Recklinghausen's disease with neurofibromas $(n=1)$, arteriovenous malformation compressing the sylvian aqueduct causing aqueductal stenosis $(n=1)$, and basal meningeal reaction due to earlier sarcoidosis $(n=1)$.

The patients with SAE (13 men and six women) were 76 (4) years old (mean (SD)) (range 67 to 84 years). The mean height was 174 (SD 7) $\mathrm{cm}$ and weight 79 (SD 12) $\mathrm{kg}$. The mean duration of symptoms was 32 (SD 25) months (table 1).

Symptoms and signs were registered semiquantitatively according to Larsson et $a l,{ }^{30}$ and Blomsterwall et al. ${ }^{31}$ Twelve clinical variables were recorded preoperatively and at the control examination performed in all patients with $\mathrm{NPH} 3$ months after shunt surgery. Indices reflecting global, psychometric, balance, gait, and continence performance were calculated as shown in table $2 .^{30}$ The psychometric tests used were the identical forms test (perceptual speed and accuracy), the reaction time test (perceptual speed and accuracy) and Bingley's visual recognition test (learning and memory). Bingley's test is done by presenting a picture of 12 drawings of familiar objects for 30 seconds. Recognition is tested immediately. The mean of two tests is used as the score. Psychiatric symptoms such as impairment of wakefulness, asteno-emotional disorder, and emotionalmotivational disorder were registered according to Lindqvist and Malmgren, ${ }^{32}$ on a four step scale (none, slight, moderate, and severe).

\section{BRAIN IMAGING}

All patients were examined by MRI except two patients with NPH and one patient with SAE, who were examined by CT due to contraindication to MRI or inability to cooperate with an MRI study. Radionuclide cisternography was carried out in all patients using [ $\left.{ }^{99 \mathrm{~m}} \mathrm{Tc}\right]-$ diethylene-triamine-penta-acetic acid (DTPA) and conventional planar imaging; $\mathrm{rCBF}$ was measured with single photon emission computed tomography (SPECT) and $\left[{ }^{99 \mathrm{~m}} \mathrm{Tc}\right]-\mathrm{HMPAO} .{ }^{28}$ The 3 month postoperative control investigation included all preoperative examinations except radionyclide cisternography. 


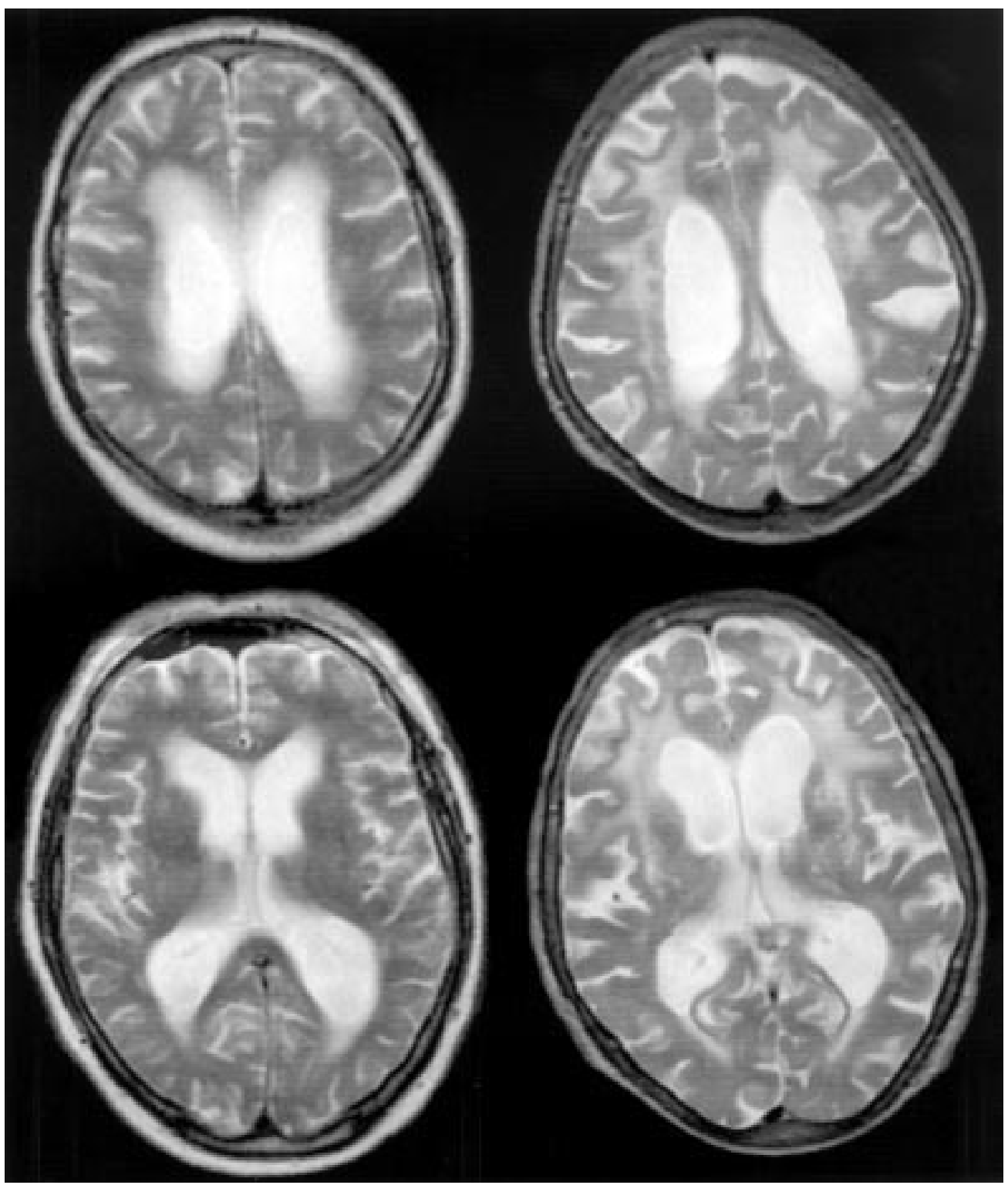

Figure 1 MRI of a patient with NPH (left) and a patient with SAE (right).

Table 1 Clinical and demographic features of NPH and SAE patients

\begin{tabular}{|c|c|c|c|}
\hline & & $\begin{array}{l}\text { NPH }(n=43) \\
\text { Mean }(S D), \\
\text { frequency }\end{array}$ & $\begin{array}{l}S A E(n=19) \\
\text { Mean }(S D) \text {, } \\
\text { frequency }\end{array}$ \\
\hline Age & & $66(12)^{\star \star \star}$ & $76(4$ \\
\hline Male/ female & & $30 / 13$ & $13 / 6$ \\
\hline Weight (kg) & & $78(17)$ & $79(12)$ \\
\hline Height $(\mathrm{cm})$ & & $173(11)$ & $174(7)$ \\
\hline Duration of symptoms (months) & & $28(29)$ & $32(25)$ \\
\hline Daily sleeping time $(\mathrm{h})$ & & $9.5(2.1)$ & $9.5(2.8)$ \\
\hline MMSE (max 30) & & $22.3(6.9)$ & $22.1(7.3)$ \\
\hline Reaction time (s) & & $0.40(0.26)$ & $0.39(0.21)$ \\
\hline Identical forms test $(\max 60)$ & & $14.2(14.8)$ & $11.5(9.8)$ \\
\hline Bingley (max 12 ) & & $3.3(2.3)$ & $3.1(2.2)$ \\
\hline IW & $(0 / 1 / 2 / 3) \dagger$ & $19 / 18 / 4 / 2^{\star}$ & $14 / 4 / 0 / 0$ \\
\hline $\mathrm{AE}$ & $(0 / 1 / 2 / 3) \dagger$ & $15 / 21 / 7 / 0$ & $10 / 5 / 3 / 0$ \\
\hline EM & $(0 / 1 / 2 / 3) \dagger$ & $25 / 16 / 2 / 0^{\star}$ & $6 / 9 / 2 / 1$ \\
\hline Urgency & (yes/no) & $22 / 21$ & $8 / 11$ \\
\hline Gait & $(1 / 2 / 3 / 4 / 5 / 6) \ddagger$ & $6 / 23 / 1 / 2 / 7 / 4$ & $1 / 10 / 3 / 2 / 2 / 1$ \\
\hline Time to walk $10 \mathrm{~m}(\mathrm{~s})$ & & $27.4(33.7)$ & $21.1(9.6)$ \\
\hline Steps to walk $10 \mathrm{~m}$ (n) & & $33(29)$ & $30(10)$ \\
\hline Diabetes & (yes/no) & $3 / 40$ & $2 / 17$ \\
\hline Hypertension & (yes/no) & $12 / 31$ & $13 / 6$ \\
\hline Cardiac disorder & (yes/no) & $12 / 31$ & $7 / 12$ \\
\hline
\end{tabular}

${ }^{\star} \mathrm{p}<0.05 ;{ }^{\star \star \star} \mathrm{p}<0.001$.

$\dagger 0-3=$ not present, slightly present, moderately present, severely present. $\ddagger 1-6=$ normal, insecure, insecure cane, bimanual support, aided, wheelchair.

$\mathrm{IW}=\mathrm{Impairment}$ of wakefulness; $\mathrm{AE}=$ astenoemotional disorder; $\mathrm{EM}=$ emotional-motivational disorder.
ANALYSIS OF CSF

Lumbar puncture was performed in the morning with the patients in a recumbent position in the L3/L4 or L4/L5 interspace. The CSF pressure was measured and the first $12 \mathrm{ml} \mathrm{CSF}$ was collected in ice chilled tubes, aliquoted, centrifuged, and frozen at $-80^{\circ} \mathrm{C}$ until analysed.

The CSF concentrations of the major monoamine metabolites homovanillic acid (HVA), 5-hydroxy-indoleacetic acid (5HIAA), and 4-hydroxy-3-methoxyphenylglycol (HMPG) were determined by high performance liquid chromatography with electrochemical detection. ${ }^{33}$ The concentration of sulfatide in CSF was determined by an immunoaffinity procedure (thin layer chromatography B immunostaining) as previously described in detail by Davidsson et $a l^{34}$ and Fredman $e t a l^{35}$ The sensitivity of the assay is 5 $\mathrm{nmol} / \mathrm{l}$ but values are given in intervals of 50 $\mathrm{nmol} / \mathrm{l}$. The within series variation of sulfatide $(\mathrm{CV})$ is $13 \%(\mathrm{n}=15)$. 
Table 2 The five functional ability indices and the respective clinical variables

\begin{tabular}{lll}
\hline Index & Function/test & Scale \\
\hline Global & MMSE (mini mental state examination) & $0-30$ \\
Psychometric & Identical forms test & $0-60$ \\
& Bingley's visual recognition test & $0-12$ \\
Balance & Reaction time test & Exact time in seconds \\
Gait & Romberg's test & $0-60$ \\
& Gait & $1-6 \dagger$ \\
Continence & Walking 10 m (time needed) & Exact time in seconds \\
& Walking 10 m (number of steps needed) & Number of steps \\
\hline
\end{tabular}

^ $1-2=$ Not present or present; $\dagger 1-6=$ normal, insecure, insecure cane, bimanual support, aided, wheelchair.

Table 3 Concentrations of sulfatide and VIP and the results of shunt surgery in patients with different aetiologies

\begin{tabular}{|c|c|c|c|}
\hline \multirow[b]{2}{*}{ Aetiology } & \multicolumn{3}{|l|}{ NPH patients } \\
\hline & $\begin{array}{l}\text { Sulfatide nmol/l } \\
\text { (Mean (SD)) }\end{array}$ & $\begin{array}{l}\text { VIP pmol/l } \\
(\text { Mean }(S D))\end{array}$ & $\begin{array}{l}\text { Overall result of } \\
\text { shunt surgery MoD } \\
\text { (Mean }(S D))\end{array}$ \\
\hline Idiopathic $(\mathrm{n}=21)$ & $203(91)$ & $8.4(4.9)$ & $0.51(0.57)$ \\
\hline Subarachnoid haemorrhage $(n=9)$ & $164(128)$ & $5.2(0.4)$ & $0.83(0.94)$ \\
\hline Cerebrovascular disease $(n=5)$ & $288(85)$ & $9.0(0.0)$ & $0.09(0.40)$ \\
\hline Trauma $(n=4)$ & $217(76)$ & $6.5(2.1)$ & $0.48(0.5)$ \\
\hline Other $(n=4)$ & $200(100)$ & $6.0(1.4)$ & $0.55(0.61)$ \\
\hline All $(n=43)$ & $206(99)$ & $7.3(3.7)$ & $0.53(0.65)$ \\
\hline
\end{tabular}

$\mathrm{MoD}=$ Mean of differences.

Table 4

\begin{tabular}{|c|c|c|c|c|}
\hline & $\begin{array}{l}\text { NPH patients } \\
(\text { Mean }(S D))\end{array}$ & $\begin{array}{l}\text { SAE patients } \\
(\text { Mean }(S D))\end{array}$ & $\begin{array}{l}\text { Laboratory } \\
\text { reference values } \\
((S D))\end{array}$ & \\
\hline Albumin ratio & $9.5(7.4)$ & $11.3(7.0)$ & $<10$ & \\
\hline \multicolumn{5}{|l|}{ Transmitter function: } \\
\hline $\mathrm{HVA}(\mathrm{nmol} / \mathrm{l})$ & $215 \quad(145)$ & $247 \quad(108)$ & $253(109)$ & $\mathrm{n}=114^{33}$ \\
\hline 5-HIAA (nmol/l) & 113 (75) & $148(52)^{\star}$ & $125(54)$ & $\mathrm{n}=114^{33}$ \\
\hline $\mathrm{HMPG}(\mathrm{nmol} / \mathrm{l})$ & $37 \quad(16)$ & 39 (8) & $47(10)$ & $\mathrm{n}=114^{33}$ \\
\hline $\mathrm{NPY}(\mathrm{pmol} / \mathrm{l})$ & 101 (33) & $117(15)^{\star \star}$ & $120-170^{36}$ & \\
\hline VIP (pmol/1) & $7.3(3.7)$ & $8.9(2.4)^{\star \star}$ & $<20^{37}$ & \\
\hline GABA (nmol/l) & $67 \quad(35)$ & $79 \quad(25)^{\star}$ & $50-130^{40}$ & \\
\hline \multicolumn{5}{|l|}{ Demyelination: } \\
\hline Sulfatide $(\mathrm{nmol} / \mathrm{l})$ & $206 \quad(99)$ & $766 \quad(957)^{\star \star \star}$ & $<300^{33}$ & \\
\hline \multicolumn{5}{|l|}{ Gliosis: } \\
\hline GD3 (nmol/1) & $38 \quad(23)$ & $(11)$ & $50(12)$ & $\mathrm{n}=101^{39}$ \\
\hline $\mathrm{GFAP}(\mathrm{ng} / \mathrm{l})$ & 1492 (950) & $(765)$ & $<750^{41}$ & \\
\hline $\mathrm{S} 100(\mu \mathrm{g} / \mathrm{l})$ & $2.7(0.9)$ & $2.6(0.8)$ & $<5$ & \\
\hline \multicolumn{5}{|l|}{ Neuronal degeneration: } \\
\hline NFL (ng/l) & $2252 \quad(4084)$ & $(704)$ & $<300^{42}$ & \\
\hline TAU (ng/l) & 265 (255) & 139 (78) & $<400^{38}$ & \\
\hline NSE $(\mu \mathrm{g} / \mathrm{l})$ & $5.2(3.3)$ & $4.4(1.5)$ & $<10.5$ & \\
\hline
\end{tabular}

${ }^{\star} \mathrm{p}<0.05 ;{ }^{\star \star} \mathrm{p}<0.01 ;{ }^{\star \star \star} \mathrm{p}<0.001$. Superscript numbers are literature references to laboratory reference values.

Neuropeptide Y (NPY) ${ }^{36}$ and vasoactive intestinal peptide (VIP) $)^{37}$ were determined as previously described. Tau protein was determined using a sandwich enzyme linked immunosorbent assay (ELISA) constructed to measure total tau (both normal tau and PHFtau), as described previously. ${ }^{38}$ Ganglioside GD3 was determined as previously described. ${ }^{39}$ $\gamma$-Aminobutyric acid (GABA) was determined by a modification of the procedure described by Goldsmith et al. ${ }^{40}$ The $o$-phtalaldehyde derivatives of GABA were analysed by reversed phase liquid chromatography with fluorescence detection. Neurofilament protein (NFL) ${ }^{22} 41$ and glial fibrillary acidic protein (GFAP) ${ }^{22} 42$ were determined as previously described. Neuron specific enolase and S-100 protein were determined according to the instructions of the manufacturer (AB Sangtec medical, Bromma, Sweden).

The study was approved by the Göteborg University ethics committee.
STATISTICS

Simple descriptive statistics were carried out using univariate analysis. Non-parametric one way analysis of variance (ANOVA) and Wilcoxon's rank sum test were used in group comparisons and the Kruskal-Wallis test when comparing more than two groups (SAS Institute Inc software). Spearman's rank test was used in correlation analysis. Adjustment for age was based on Mantel's technique of pooling ${ }^{43}$ applied to Fisher's non-parametric permutation test. ${ }^{44}$ No correction for multiple correlations was made. The indices were constructed by calculating the mean of the variables included in each index (table 2). Standardisation was used to enable comparison of scores. ${ }^{30}$ The preoperative variables were standardised to a mean of 0 and an $\mathrm{SD}$ of 1 . In the postoperative statistics, the mean and SD of the preoperative variables were used in the standardisation calculation. The difference in each index between preoperative and postoperative value was calculated, and the mean of the differences (MoD) between the preoperative and postoperative indices in each patient was calculated as the overall result after shunt surgery. Values are listed as mean (SD).

\section{Results}

Thirty four $(87 \%)$ of the patients with $\mathrm{NPH}$ improved after shunt surgery (MoD $>0.10$, range 0.10-2.2), two were unchanged $(\mathrm{MoD}=0.01$ and 0.09$)$, and three deteriorated $(\mathrm{MoD}=-0.38,-0.49$, and -0.94$)$ (table 3 ). Four patients with NPH refused to participate in the 3 month postoperative examination but were all reported as improved. The patients with NPH were 10 years younger than the patients with SAE and clinically characterised by more impairment of wakefulness and less emotional motivational symptoms. No other differences in clinical variables were found (table 1).

Significant differences between the patients with SAE and those with NPH were found for sulfatide, VIP, NPY, 5-HIAA, and GABA (table 4).

Most apparent was the increase of sulfatide in patients with SAE. With a cut off level of 400 nmol/1 sulfatide (mean of $\mathrm{NPH}+2 \mathrm{SD}$ ), the patients with SAE and those with NPH could be separated with a sensitivity of $74 \%$ and a specificity of $94 \%$ (fig 2). Patients with SAE had higher CSF sulfatide than the subgroup of patients with idiopathic NPH $(p<0.0001)$. One of the two patients in the NPH group with the highest sulfatide concentrations $(400 \mathrm{nmol} / \mathrm{l})$ had hypertension, cerebrovascular disease, and diabetes and the other had had a previous subarachnoidal haemorrhage. Both of them improved after shunt surgery. The improvement of the patients with NPH with cerebrovascular aetiology was less pronounced than in any other group $(\mathrm{p}<0.01)$ whereas CSF sulfatide was found to be higher at trend level $(p=0.09)$ in this group compared with the other patients with NPH (table 3). Sulfatide values in CSF for four of the five patients with NPH with cerebrovascular aetiology were 200, 250, 300, and $400 \mathrm{nmol} / \mathrm{l}$. There was no correlation between CSF sulfatide and total outcome after shunt 
CSF sulfatide in NPH and SAE patients

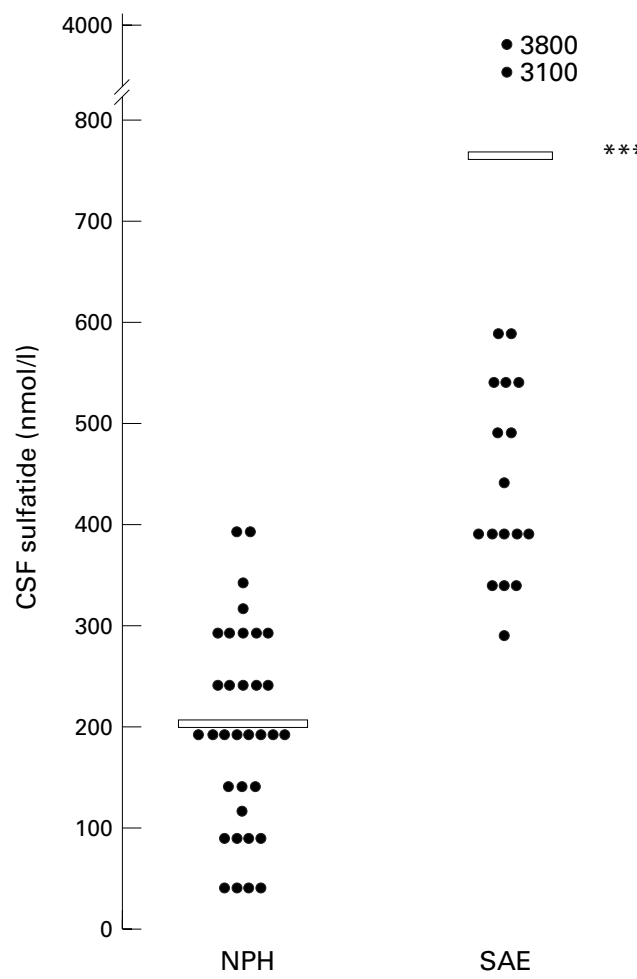

Figure 2 Sulfatide concentrations in NPH and patients with SAE. Mean concentrations are indicated by lines. Wilcoxon rank sum test.

surgery in patients with NPH. The two patients with SAE with exceptionally high concentrations of CSF sulfatide (3800 and $3100 \mathrm{nmol} / \mathrm{l}$ ) had no signs of blood-brain barrier dysfunction or immunopathy and they were not clinically different from the other patients with SAE. After adjustment for age, the significant difference in sulfatide between patients with NPH and those with SAE remained.

VIP in CSF was slightly but significantly higher in patients with SAE than in patients with NPH $(p<0.01)$, but each patient was still within the normal range. VIP was higher in patients with NPH with cerebrovascular aetiology than in the other patients with NPH $(p<0.05)$ (table 3). A high VIP correlated with a poor total outcome after shunt surgery $(r=-0.54)$.

5-HIAA was higher in patients with SAE and correlated with duration of symptoms in the whole material of NPH and patients with SAE $(r=-0.30, \mathrm{p}<0.05)$.

No correlation between results of shunt surgery, height, weight, and duration of symptoms and CSF concentrations of GABA and NPY was seen.

NFL and GFAP in CSF were increased in both patients with NPH and those with SAE compared with reference values but no difference was found between the two disorders.

Routine CSF analyses showed no differences between the groups for number of erythrocytes, mononuclear and polynuclear cells, albumin ratio (CSF albumin $\times 10^{3} /$ serum albu$\mathrm{min}$ ), immunoglobulin-G (IgG) index (CSF $\mathrm{IgG} /$ serum IgG divided by albumin ratio) and presence or absence of immunopathy. In the whole material, nine patients had more than 100 erythrocytes $/ \mathrm{mm}^{3}$, five patients had more than 1 polynuclear cell $/ \mathrm{mm}^{3}$, and five patients had more than 5 mononuclear cells $/ \mathrm{mm}^{3}$. Four patients had an increased IgG index. Those patients with bleeding into the subarachnoidal space (increased number of CSF erythrocytes), increased numbers of polynuclear or mononuclear cells, or increased IgG index were not different from those with a normal cell count for clinical or other CSF variables. Exclusion of patients with an abnormal cell count had no effect on the results reported below. The three patients with aqueductal stenosis had higher GABA $(p<0.05)$ and lower NSE $(p<0.05)$ compared with other patients with NPH. Exclusion of these three patients had no effect on the results reported.

Patient's height correlated negatively with 5-HIAA $(r=-0.44)$ and HVA $(r=-0.39$; $\mathrm{p}<0.01)$, weight positively with VIP $(r=0.43$, $\mathrm{p}<0.05)$, and age positively with sulfatide $(r=0.44, \mathrm{p}<0.001)$.Concentrations of HMPG were higher in men than in women $(\mathrm{p}<0.05)$. Patients with cardiovascular disease had higher sulfatide concentrations than those without $(p<0.05)$. No differences in any CSF variable were seen between patients with and without diabetes and hypertension.

\section{Discussion}

The white matter degeneration in SAE is caused by a microangiopathy with demyelination and small vessel associated lacunae. ${ }^{16-18}$ The pathogenesis of the white matter disease in $\mathrm{NPH}$ is different from that in SAE, and characterised by oedema, gliosis, and neuronal degeneration. ${ }^{58}$ Despite the difference in pathogenesis, the changes in white matter in the two disorders can sometimes be difficult to distinguish on MRI or CT. Both disorders present with a subcortical and sometimes similar symptomatology with diagnostic and therapeutic difficulties as a consequence.

In the present study, we found that carefully selected patients with SAE, presenting with typical clinical symptoms and signs and characteristic MRI changes, had high concentrations of CSF sulfatide, a biochemical marker of demyelination. ${ }^{35}$ Sulfatide is the major acidic glycosphingolipid in myelin of the central as well as the peripheral nervous system ${ }^{45}$ and constitutes about $5 \mathrm{~mol} \%$ of the total lipids in human brain myelin. ${ }^{45}$ In the inherited leukodystrophy caused by impaired lysosomal degradation of sulfatide (metachromatic leukodystrophy) the concentration of sulfatide in CSF is increased. ${ }^{47}$ Sulfatide is also increased in CSF from patients with vascular dementia. ${ }^{35}$ Evidence of recent or ongoing major ischaemic events was not seen in any of our patients with SAE, clinically or at MRI, so that the increased sulfatide concentration seen in all the patients with SAE indicates that the demyelination is an ongoing process with continuous release of myelin constituents to the CSF. Demyelination secondary to regressive changes in the astroglia, activation of the microglia, and decrease of oligodendrocytes have been shown in chronic 
states of ischaemia in the deep white matter in patients with SAE. ${ }^{16}$ The continuous release of myelin constituents to the CSF is consistent with the results of Pantoni et $a l^{48}$ showing that the progression of symptoms in SAE is continuous rather than stepwise.

In most of our patients with $\mathrm{NPH}$, sulfatide in CSF was normal, indicating that demyelination, as seen in SAE, plays a minor or no part in the pathophysiology of NPH. A few patients with NPH had increased sulfatide and there was a slight overlap of CSF sulfatide in the range of 300 to $400 \mathrm{nmol} / \mathrm{l}$ between patients with SAE and NPH. We think that the most probable explanation would be the presence of irreversible ischaemic white matter lesions in some patients with NPH. This assumption is supported not only by the fact that the patients with NPH with cerebrovascular aetiology had higher sulfatide concentrations than other patients with NPH at trend level, but also by the finding that the improvement after shunt surgery was less pronounced in this group. Coexistence of cerebrovascular disorders and $\mathrm{NPH}$ was also reported by Bech et $a l,{ }^{49}$ who found that some of their patients with NPH had parenchymal pathoanatomical changes typical of vascular dementia and Alzheimer's disease. Clinical studies have also shown that NPH and SAE often coexist in a single patient. $^{20} 21$ Furthermore, MRI studies have shown that deep white matter lesions associated with vascular disease are common in $\mathrm{NPH},{ }^{10}{ }^{12}$ further stressing the existence of an overlap between the two disease entities.

Despite this overlap, one of the main results in this study is the finding of increased CSF sulfatide concentrations in patients with SAE and the high sensitivity (74\%) and specificity (94\%) with which this assay can discriminate $\mathrm{NPH}$ from SAE, making analysis of this marker an important tool in the diagnostic and pathophysiological work in the field.

The increase in GFAP concentrations in the patients with NPH is in accordance with previous reports and probably reflects the gliotic process in the periventricular tissue. ${ }^{22} 50 \mathrm{We}$ found no indications of astroglial cell disintegration as the CSF concentration of S-100 protein was within normal limits, thus further supporting the conclusion that the increased concentration of GFAP is due to a gliosis in the periventricular tissue. We recently reported that NFL, a marker of axonal degeneration, was increased in patients with NPH, indicating ongoing but still reversible axonal damage in $\mathrm{NPH} .{ }^{22}$ The same result was obtained in the present study, strongly indicating that axonal dysfunction is an important feature in the pathogenesis of NPH. There was also an increase of NFL in patients with SAE, but not to the same extent as in NPH. A similar result was recently obtained in another study on vascular dementia, probably reflecting the subcortical white matter disease in this disorder. ${ }^{51}$

A second important result of this study is that patients with NPH are characterised by high NFL but normal sulfatide concentrations in CSF. This may be compatible with a disturbance of axonal transmission without signs of demyelination. NPH is possibly a disorder with intact myelin sheaths but with a disturbed axonal function with continuous release of neuronal structural elements such as NFL, which is the essential component of the neurofilament core. The symptoms of NPH can be reversed by lumbar spinal drainage, ${ }^{27}$ further indicating that the biochemical disturbance in NPH must be reversible within hours, excluding demyelination as a more important process. The patients with SAE also showed an increase of NFL but not to the same extent as in NPH, indicating an axonal degenerative process in this disorder. It should be emphasised that both of these processes are involved in both $\mathrm{NPH}$ and SAE. However, our results indicate that the two pathophysiological mechanisms differ in intensity. Furthermore, the known negative correlation between long duration and reversibility of symptoms in NPH also indicate that a wallerian degenerative and irreversible process is present in NPH. The combined use of CSF sulfatide and NFL might be a useful tool in predicting the degree of reversibility in patients with NPH with signs and symptoms of cerebrovascular disorders. This should be an important question to answer in future research.

The CSF concentration of tau has been suggested to reflect the degree of neuronal, preferentially axonal, degeneration. ${ }^{38}$ However, high concentrations are found primarily in disorders with cortical degeneration. A moderate increase in CSF tau is found in Alzheimer's disease, ${ }^{38}$ and in Creutzfeldt-Jakob's disease with a very intense cortical neuronal degeneration CSF tau is markedly increased. ${ }^{52}$ In the present study, we did not find any increase in CSF tau in either NPH or SAE, both of which are characterised by subcortical white matter degeneration. These findings suggest that there is only minor cortical degeneration in $\mathrm{NPH}$ and SAE, and that analysis of CSF tau may aid in the differentiation of these disorders from Alzheimer's disease.

A typical clinical feature of NPH is impairment of wakefulness. ${ }^{32}$ A possible explanation could be decreased activity in the ascending monoaminergic neurons connecting to the thalamus, hippocampus, and basal frontal cortex. Malm et al did not find any changes in CSF monoamine metabolites in patients with NPH. ${ }^{53}$ We found lower CSF 5-HIAA concentrations in NPH than in SAE, which might suggest a reduced serotoninergic transmission in NPH compatible with a down regulation of brain stem function in NPH.

VIP is the most potent dilator of the cerebrovascular system. Previous reports show that VIP in CSF is reduced in NPH compared with controls and that concentrations increase after shunt surgery, ${ }^{37}{ }^{54}$ implying reduced VIP transmission in NPH secondary to the reduced metabolism and $\mathrm{rCBF}^{28}{ }^{29} \mathrm{~A}$ speculative explanation for the higher VIP concentration in patients with SAE could be that the VIP-ergic neurons are activated to accomplish a compensatory vasodilatation. This notion is supported by the fact that patients with cerebrovascular 
$\mathrm{NPH}$ had higher VIP concentrations than those with other aetiologies.

It could be argued that the differences in sulfatide might be due to altered CSF dynamics in NPH with reversed CSF flow into the periventricular tissue. ${ }^{3-6}$ If this were the case, similar differences in other variables reflecting axonal degeneration or gliosis would be expected. However, these variables were higher in NPH than in SAE, suggesting that the differences reflect true pathophysiological changes and not CSF dynamic changes. Further, no differences in HVA and HMPG were found whereas 5-HIAA was reduced in $\mathrm{NPH}$, also contradicting CSF dynamics as an explanation for the differences. Moreover, CSF sulfatide was higher in patients with $\mathrm{NPH}$ with cerebrovascular aetiology than in other NPH subgroups, which could not be explained by alterations of CSF dynamics.

The patients with NPH and those with SAE were well matched except for age. The patients with SAE were carefully selected, all presenting with typical signs and symptoms of microvascular lesions. No patients with large vessel disease (multiple cortical infarctions) at MRI were included. All patients therefore meet the most specific clinical criteria for SAE. Our rigid inclusion criteria are a probable explanation for the pronounced difference in sulfatide between NPH and patients with SAE. It could be argued that the results reported would have been even more reliable if the SAE diagnosis had been confirmed pathoanatomically at necropsy but we have no reason to think that any of the patients with SAE included were misdiagnosed. Our diagnostic criteria seem appropriate and sufficient for identification of ischaemic lesions in SAE as all these patients had increased concentrations of CSF sulfatide.

The diagnostic criteria used for patients with $\mathrm{NPH}$ are valid as $87 \%$ of them improved after shunt surgery. The diagnostic and predictive problem in NPH is the cerebrovascular disease subgroup and the known coexistence of NPH and SAE.

In conclusion, the most important results of this study are:

- CSF sulfatide, a marker of demyelination, is increased in SAE and distinguishes between SAE and patients with NPH with a sensitivity of $74 \%$ and a specificity of $94 \%$, making it an important diagnostic tool.

- The overlap in CSF sulfatide between $\mathrm{NPH}$ and patients with SAE is small and probably explained by the coexistence of cerebrovascular disorder in a subgroup of patients with NPH.

Patients with NPH are characterised by increased NFL and normal sulfatide in the CSF, possibly reflecting a state of reversible axonal degeneration and no structural demyelination.

This study was supported by research grants from the Edit Jacobson Foundation, the John and Brit Wennerström Foundation, the Hjalmar Svensson Foundation, the Rune and Ulla Ahlmlöv Foundation, the Göteborg Medical Society, Landstingsgemensamt FoU-råd (Bohuslandstinget, Sweden), the ingsgemensamt FoU-råd (Bohuslandstinget, Sweden), the
Swedish Association of Neurologically Disabled, and the Swedish Medical Research Council (Grants 11560 and 12103).
1 Adams RD, Fisher CM, Hakim S, et al. Symptomatic occult hydrocephalus with normal cerebrospinal-fluid pressure. A treatable syndrome. N Engl f Med 1965;273:117-26.

2 Hakim S, Adams RD. The special clinical problem of symptomatic hydrocephalus with normal cerebrospinal fluid pressure. Observations on cerebrospinal fluid hydrodynamics. $\mathcal{F}$ Neurol Sci 1965;2:307-27.

3 Milhorat TH, Clark RG, Hammock MK, et al. Structural, ultrastructural, and permeability changes in the ependyma and surrounding brain favoring equilibration in progressive hydrocephalus. Arch Neurol 1970;22:397-407.

4 Milhorat TH. The third circulation revisited. $f$ Neurosurg 1975;42:628-45.

5 Miyagami M, Shibuya T, Tsubokawa T. Subependymal CSF absorption in hydrocephalic oedema. In: Matsumoto $\mathrm{S}$, and Tamaki N, eds. Hydrocephalus, pathogenesis and treatment. Tokyo: Springer Verlag, 1981:181-94.

6 Deo Narine V, Gomez DG, Vullo T, et al. Direct in vivo observation of transventricular absorption in the hydrocephalic dog using magnetic resonance imaging. Invest Radiol 1994;29:287-93.

7 Weller RO, Wisniewski H, Shulman K. Experimental hydrocephalus in young dogs. I Neuropathol Exp Neurol 1971;30:613-26.

8 Akai K, Uchigasaki S, Tanaka U, et al. Normal pressure hydrocephalus. Neuropathological study. Acta Pathologica faponicum 1987;37:97-110.

9 Godersky JC, Graff Radford NR, Yuh WT. Magnetic resonance imaging of patients with normal pressure hydrocephalus. Adv Neurol 1990;52:554.

10 Bradley Jr WG, Whittemore AR, Watanabe AS, et al. Association of deep white matter infarction with chronic communicating hydrocephalus: implications regarding the possible origin of normal-pressure hydrocephalus [see comments]. Am $\mathcal{F}$ Neuroradiol 1991;12:31-9.

11 Davis PC, Mirra SS, Alazraki N. The brain in older persons with and without dementia: findings on MR, PET, and SPECT images. Am ₹ Roentgenol 1994;162:1267-78.

12 Krauss JK, Droste DW, Vach W, et al. Cerebrospinal fluid shunting in idiopathic normal-pressure hydrocephalus of shunting in idiopathic normal-pressure hydrocephalus of lesions. Neurosurgery 1996;39:292-9.

13 Babikian V, Ropper AH. Binswanger's disease: a review. Stroke 1987;18:2-12

14 Bennett DA, Wilson RS, Gilley DW, et al. Clinical diagnosis of Binswanger's disease. $\mathcal{F}$ Neurol Neurosurg Psychiatry 1990;53:961-5.

15 Pantoni L, Garcia JH. The significance of cerebral white matter abnormalities 100 years after Binswanger's report. A review. Stroke 1995;26:1293-301.

16 Akiguchi I, Tomimoto H, Suenaga T, et al. Alterations in glia and axons in the brains of Binswanger's disease patients. Stroke 1997;28:1423-9.

17 Zhang WW, Olsson Y. The angiopathy of subcortical arteriosclerotic encephalopathy (Binswanger's disease): mmunohistochemical studies using markers for components of extracellular matrix, smooth muscle actin and endothelial cells. Acta Neuropathol (Berl) 1997;93:219-24.

18 Brun A. Pathology and pathophysiology of cerebrovascular dementia: pure subgroups of obstructive and hypoperfusive etiology. Dementia 1994;5:145-7.

19 Bracco L, Campani D, Baratti E, et al. Relation between MRI features and dementia in cerebrovascular disease patients with leukoaraiosis: a longitudinal study. $f$ Neurol Sci 1993;120:131-6.

20 Graff Radford NR, Godersky JC. Idiopathic normal pressure hydrocephalus and systemic hypertension. Neurolpressure hydrocepha
ogy $1987 ; 37: 868-71$

21 Koto A, Rosenburg G, Zingesser LH, et al. Syndrome of normal pressure hydrocephalus: possible relation to hypertensive and arteriosclerotic vasculopathy. $\mathcal{F}$ Neurol Neurosurg Psychiatry 1977;40:73-9.

22 Tullberg $M$, Rosengren L, Blomsterwall E, et al. CSF neurofilament and glial fibrillary acidic protein in normal pressure hydrocephalus. Neurology 1998;50:1122-7.

23 Sutton LN, Wood JH, Brooks BR, et al. Cerebrospinal fluid myelin basic protein in hydrocephalus. $f$ Neurosurg 1983;59:467-70

24 Skoog I, Wallin A, Fredman P, et al. A population study on blood-brain barrier function in 85-year-olds: relation to Alzheimer's disease and vascular dementia. Neurology 1998;50:966-71.

25 Wikkelso C, Blomstrand C. Cerebrospinal fluid proteins and cells in normal-pressure hydrocephalus. $\mathcal{F}$ Neurol 1982; 228: $171-80$.

26 Larsson A, Moonen M, Bergh AC, et al. Predictive value of quantitative cisternography in normal pressure hydrocephalus. Acta Neurol Scand 1990;81:327-32.

27 Wikkelso C, Andersson H, Blomstrand C, et al. Normal pressure hydrocephalus. Predictive value of the cerebrospinal fluid tap-test. Acta Neurol Scand 1986;73:566-73.

28 Larsson A, Bergh AC, Bilting M, et al. Regional cerebral blood flow in normal pressure hydrocephalus: diagnostic and prognostic aspects. Eur F Nucl Med 1994;21:118-23.

29 Graff Radford NR, Rezai K, Godersky JC, et al. Regional cerebral blood flow in normal pressure hydrocephalus. $\mathcal{F}$ Neurol Neurosurg Psychiatry 1987;50:1589-96.

30 Larsson A, Wikkelso C, Bilting M, et al. Clinical parameters in 74 consecutive patients shunt operated for normal presin 74 consecutive patients shunt operated for normal pres-

31 Blomsterwall E, Bilting M, Stephensen H, et al. Gait abnormality is not the only motor disturbance in normal pressur hydrocephalus. Scand F Rehabil Med 1995;27:205-9. 
32 Lindqvist $\mathrm{G}$, Andersson $\mathrm{H}$, Bilting $\mathrm{M}$, et al. Normal pressure hydrocephalus: psychiatric findings before and after shun operation classified in a new diagnostic system for organ

psychiatry. Acta Psychiatr Scand Suppl 1993;373:18-32.
33 Blennow K, Wallin A, Gottfries CG, et al. Concentration graBlennow K, Wallin A, Gottfries CG, et al. Concentration gradients for monoamine metabolites in lumbar cerebrospinal
fluid. 7 Neural Transm Park Dis Dement Sect 1993;5:5-15.

34 Davidsson P, Fredman P, Mansson JE, et al. Determination of gangliosides and sulfatide in human cerebrospinal fluid with a microimmunoaffinity technique. Clin Chim Acto 1991;197:105-15.

35 Fredman P, Wallin A, Blennow $\mathrm{K}$, et al. Sulfatide as a biochemical marker in cerebrospinal fluid of patients with vascular dementia. Acta Neurol Scand 1992;85:103-6.

36 Widerlov E, Lindstrom LH, Wahlestedt C, et al. Neuropeptide $\mathrm{Y}$ and peptide YY as possible cerebrospinal fluid markers for major depression and schizophrenia, respectively. F Psychiatr Res 1988;22:69-79.

37 Wikkelso C, Ekman R, Westergren I, et al. Neuropeptides in cerebrospinal fluid in normal-pressure hydrocephalus and cerebrospinal fluid in normal-pressure

38 Blennow K, Wallin A, Agren $\mathrm{H}$, et al. Tau protein in cerebrospinal fluid: a biochemical marker for axonal degeneration in Alzheimer disease? Mol Chem Neuropatho 1995;26:231-45.

39 Lekman A, Fredman P. A new procedure for determining ganglioside GD3 a potential glial cell activation marker in cerebrospinal fluid. Neurochem Int 1998;33:103-8.

40 Goldsmith RF, Earl JW, Cunningham AM. Determination of delta-aminobutyric acid and other amino acids in cerebrospinal fluid of pediatric patients by reversed-phase liquid chromatography. Clin Chem 1987;33:1736-40.

41 Rosengren LE, Karlsson JE, Karlsson JO, et al. Patients with amyotrophic lateral sclerosis and other neurodegenerative diseases have increased levels of neurofilament protein in CSF. F Neurochem 1996;67:2013-18.

42 Rosengren LE, Wikkelso C, Hagberg L. A sensitive ELISA for glial fibrillary acidic protein: application in CSF of for glial fibrillary acidic protein: applica

43 Mantel N. Chi-square test with one degree of freedom. Extension of the Mantel-Haenzel procedure. Fournal of the American Statistical Association 1963;59:690-700.
44 Bradley JV. Distribution-free statistical test. London: PrenticeHall, 1968.

45 Rosengren B, Fredman P, Månsson J-E, et al. Lysosulfatide (galactosylsphingosine-3-O-sulfate) from metachromatic leukodystrophy and normal human brain. $\mathcal{F}$ Neurochem 1989;52:1035-41.

46 Svennerholm L, Boström K, Fredman P, et al. Membrane lipids of human peripheral nerve and spinal cord. Biochim Biophys Acta 1992;1128:1-7.

47 Malm G, Ringden O, Winiarski J, et al. Clinical outcome in four children with metachromatic leukodystrophy treated by bone marrow transplantation. Bone Marrow Transplantation 1996;17:1003-8.

48 Pantoni L, Garcia JH, Brown GG. Vascular pathology in three cases of progressive cognitive deterioration. 7 Neurol Sci $1996 ; 135: 131-9$.

49 Bech RA, Juhler M, Waldemar G, et al. Frontal brain and leptomeningeal biopsy specimens correlated with cerebrospinal fluid outflow resistance and B-wave activity in patients suspected of normal-pressure hydrocephalus. Neurosurgery 1997;40:497-502.

50 Albrechtsen M, Sorensen PS, Gjerris F, et al. High cerebrospinal fluid concentration of glial fibrillary acidic protein (GFAP) in patients with normal pressure hydrocephalus. $\mathcal{F}$ Neurol Sci 1985;70:269-74.

51 Rosengren L E, Karlsson J E, Sjogren M, et al. Neurofilament protein levels in CSF are increased in dementia. Neurology 1999;23;52:1090-3.

52 Otto $\mathrm{M}$, Wiltfang J, Tumani $\mathrm{H}$, et al. Elevated levels of tauprotein in cerebrospinal fluid of patients with CreutzfeldtJakob disease. Neurosci Lett 1997;225:210-2.

53 Malm J, Kristensen B, Ekstedt J, et al. CSF monoamine metabolites, cholinesterases and lactate in the adult hydrocephalus syndrome (normal pressure hydrocephalus) related to CSF hydrodynamic parameters. $\mathcal{F}$ Neurol Neurosurg Psychiatry 1991;54:252-9.

54 Wikkelso C, Fahrenkrug J, Blomstrand C, et al. Dementia of different etiologies: vasoactive intestinal polypeptide in CSF. Neurology 1985;35:592-5. 